\title{
The Hubbard model for alternant and nonalternant systems ${ }^{a)}$
}

\author{
Stephen Lee \\ Department of Chemistry, University of Michigan, Ann Arbor, Michigan 48109
}

(Received 10 March 1988; accepted 12 October 1988)

In this paper we consider the effects of two-electron, one-center interactions when added to the one-electron, two-center molecular orbital model. There are, therefore, two parameters considered: The standard Hückel $\beta$ (two-center, one-electron) term and the Hubbard onecenter, two-electron term $U$. It is shown how the change in the ground state as one changes the $U / \beta$ ratio is highly dependent on the presence or absence of odd member rings.

\section{INTRODUCTION}

Some years ago it was fashionable to debate the relative merits of molecular orbital and valence bond theory. Since then, it has become universally acknowledged that both methods can be valid starting points for calculation. In certain cases the same chemical system may be treated by either technique.' There is no a priori reason why this should be true. For a method to be physically relevant, the true system must be located inside the circle whose epicenter is the initial trial solution and whose radius is the radius of convergence of its perturbation expansion. The molecular orbital and valence bond methods have very different starting points, each with its own quite different perturbation expansion. At first glance, they are not even in the same Hilbert space.

Nevertheless, in this paper we show that in systems which contain no odd member rings, and which preferably have low atomic coordination numbers and no squares, both molecular orbital and valence bond methods provide suitable starting points for calculation. In this paper we will use the Hückel Hamiltonian as a paradigm for molecular orbital calculations and the Heisenberg-Dirac spin Hamiltonian for valence bond calculations.

\section{DEFINITIONS}

The Hückel Hamiltonian is a one-electron Hamiltonian. It may be considered to be acting on a space of atomic orbitals. In this case:

$$
\mathbf{H}_{\text {Hücket }}=\sum_{i, j}\left|\chi_{i}\right\rangle \beta_{i j}\left\langle\chi_{j}\right|,
$$

where $\left|\chi_{i}\right\rangle$ and $\left|\chi_{j}\right\rangle$ are atomic orbitals and

$$
\beta_{i j}= \begin{cases}\beta & \text { if } \chi_{i} \text { and } \chi_{j} \text { are nearest neighbors } \\ 0 & \text { otherwise }\end{cases}
$$

Alternatively the Hückel Hamiltonian may be considered to be acting on $n$-electron wave functions (we shall call such $n$ electron wave functions SDs for Slater determinants). In this case:

$$
\mathbf{H}_{\text {Hückel }}=\sum \beta_{i j} a_{i \alpha}^{\dagger} a_{j \alpha},
$$

where $a_{i \alpha}^{\dagger}$ and $a_{i \alpha}$ are, respectively, the creation and annihilation operators for $\chi_{i \alpha}$ and where the $\alpha$ index denotes

a) Dedicated to my parents on the occasion of their sixtieth birthdays. whether the $i$ orbital is up-spin $(\alpha=+)$ or down-spin $(\alpha=-)$.

The Heisenberg-Dirac spin Hamiltonian (HD) acts only on a space of SDs.

$$
\mathbf{H}_{\mathrm{HD}}=-\sum J_{i j} \mathbf{S}_{i} \cdot \mathbf{S}_{j},
$$

where $\mathbf{S}_{i}$ and $\mathbf{S}_{j}$ are spin $-1 / 2$ operators indexed by atomic orbital and

$$
J_{i j}=\left\{\begin{array}{ll}
J & \text { if } \beta_{i j}=\beta \\
0 & \text { otherwise }
\end{array} .\right.
$$

Both Hückel and HD Hamiltonians are asymptotic cases of the Hubbard Hamiltonian ${ }^{2}$ :

$$
\mathbf{H}_{\mathrm{Hubbard}}=\sum_{\substack{i>j \\ \alpha}} \beta_{i j} a_{i \alpha}^{\dagger} a_{j \alpha}+U \sum_{i} a_{i+}^{\dagger} a_{i-}^{\dagger} a_{i-} a_{i+} .
$$

Thus when $U=0$,

$$
\mathbf{H}_{\text {Hubbard }}=\mathbf{H}_{\text {Hückel }}
$$

and when $U \gg|\beta|$,

$$
P \mathrm{H}_{\mathrm{Hubbard}} P=\mathrm{H}_{\mathrm{HD}} \text {. }
$$

Equation ( 8 ) points out the essential difference between the space of either the Hückel or the Hubbard Hamiltonian and the HD Hamiltonian. In the HD Hamiltonian, the Hamiltonian is defined only on SDs in which every spatial atomic orbital is singly occupied. $S_{i}$ can not act on an SD which has neither $\left|\chi_{i_{+}}\right\rangle$or $\left|\chi_{i_{-}}\right\rangle$. We call the restricted space of the HD Hamiltonian localized, as there is exactly one electron located at each orbital. The Hückel and Hubbard Hamiltonians act on a delocalized space where there is no restriction, besides that of the exclusion principle, as to the number of electrons which occupy a spatial atomic orbital. The $P$ in Eq. (8) refers to the projection of the Hubbard Hamiltonian onto the localized HD space.

Figure 1 illustrates the Hückel, HD, and Hubbard Hamiltonians for the $\pi$ orbitals of trimethylenemethane 1.<smiles>[O-][O+]([O-])[O-]</smiles> 
<smiles></smiles>

(a)<smiles>[14CH3][14CH3]</smiles>

ii.<smiles>[14CH3]O[14CH3]</smiles>

iii.

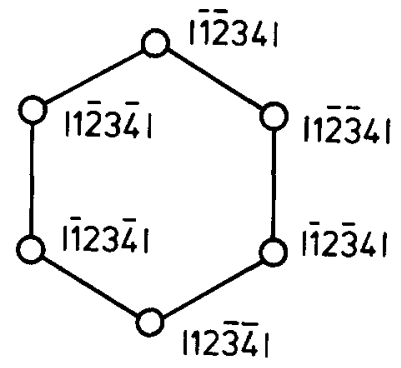

(b)

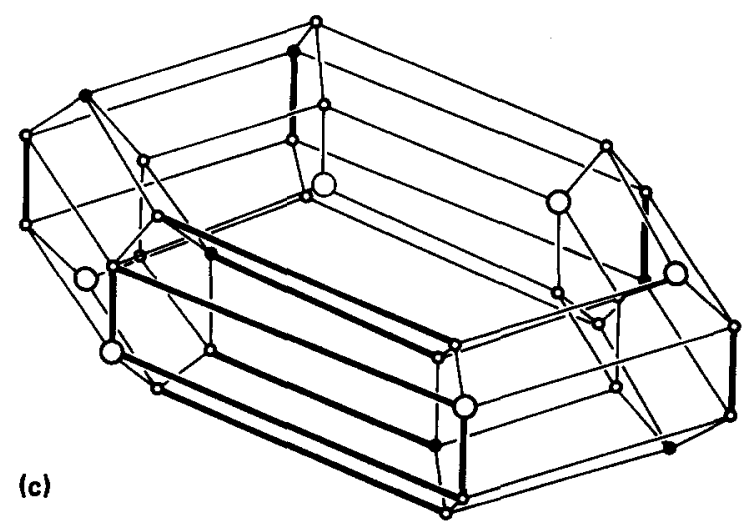

FIG. 1. The Hückel, HD, and Hubbard diagrams of 1. (a) The standard Hückel diagram. Circles represent orbitals, lines $\beta$-interactions. (b) The HD diagrams. (i) is the $S_{z}=2$ manifold, (ii) the $S_{z}=1$ and (iii) the $S_{z}$ $=0$. Open circles represent SDs. Lines are the $J$ exchange interactions. (c) The Hubbard diagram. Large open circles are localized SDs (e.g., $|12 \overline{34}|$ ), small open circles single-excited SDs (e.g., $|1 \overline{1} 2 \overline{3}|$ ), and small filled circles double-excited SDs (e.g., $|1 \overline{1} 2 \overline{2}|$ ). Thin lines represent positive off-diagonal terms, thick lines negative off-diagonal terms.
TABLE I. Key to symbols.

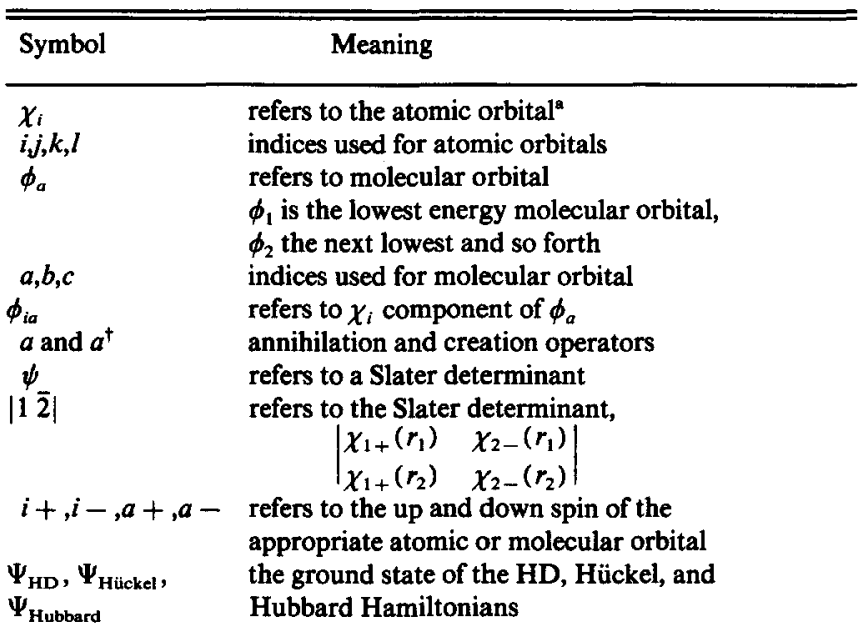

"Where no ambiguity occurs the $\chi$ label will be dropped. An example is $|1 \overline{2}|=\left|\chi_{1+} \chi_{2-}\right|$. See below.

Finally in Table I we state the notational conventions which will be used in this paper.

\section{Geometry of the HD Hamiltonlan}

We define two geometrical concepts:

(1) Alternant. ${ }^{3} \mathrm{~A}$ Hamiltonian is alternant with respect to a given basis set when the basis vectors may be divided into two sets, the starred set and the unstarred set, in such a way that all nonzero off-diagonal terms connect starred basis vectors to unstarred basis vectors.

(2) Connected. A Hamiltonian is connected with respect to a given basis set when the basis set does not create a block-diagonal Hamiltonian.

Thus the Hückel and Hubbard diagrams of Fig. 1 are both connected and alternant. Although alternant, the HD diagram of Fig. 1 is not connected.

HD and Hubbard Hamiltonians are always block diagonal, as off-diagonal matrix elements between SDs with different total directional spin quantum numbers are always zero. We may call these different blocks the $S_{z}=N / 2$, $(N / 2)-1$, etc... manifolds, where $N$ equals the total number of electrons in the system. The following lemma is evidently true:

\section{Lemma 1}

If the Hückel Hamiltonian is alternant, then both the Hubbard and HD Hamiltonians are alternant. If the Hückel Hamiltonian is connected each distinct $S_{z}$ manifold of the HD and Hubbard Hamiltonians are connected.

\section{Comparison of Huckel and HD ground states}

In this section we state three approximate results. $\mathrm{Nu}$ merical illustrations of these results are to be found in the following section.

\section{Lemma 2}

Let $\psi_{1}$ and $\psi_{2}$ be two different localized atomic SDs belonging to the same $S_{z}$ manifold. Let $p_{1}$ and $p_{2}$ be, respec- 
tively, the number of nonzero off-diagonal elements involving $\psi_{1}$ and $\psi_{2}$. If $p_{1}>p_{2}$, then generally

$$
\left.\left|\left\langle\psi_{1} \mid \Psi_{\mathrm{HD}}\right\rangle\right|\right\rangle\left|\left\langle\psi_{2} \mid \Psi_{\mathrm{HD}}\right\rangle\right|
$$

and

$$
\left|\left\langle\psi_{1} \mid \Psi_{\text {Hückel }}\right\rangle\right|>\left|\left\langle\psi_{2} \mid \Psi_{\text {Hückel }}\right\rangle\right| \text {. }
$$

\section{Proof}

One can prove this result in a variety of ways. Perhaps the simplest way is to recall that the coordination number is directly proportional to the second moment. ${ }^{4}$ If the second moment of $\psi_{1}$ is greater than that of $\psi_{2}$, then $\psi_{1}$ will in general contribute more to the most bonding eigenfunctions, and to the ground state in particular. Q.E.D.

In a similar manner one may also consider second nearest neighbors.

\section{Lemma 2a}

Let $\psi_{1}$ and $\psi_{2}$ be two different localized atomic SDs belonging to the same $S_{z}$ manifold. Let both $\psi_{1}$ and $\psi_{2}$ have the same number of first nearest neighbors (i.e., following Lemma $2, p_{1}=p_{2}$ ). Let $\psi_{1}$ though have more second nearest neighbors than $\psi_{2}$. Then, in general Eqs. (9) and (10) are also true.

\section{Proof}

The proof is identical to the previous one, except now one considers the fourth moment. Q.E.D.

\section{Lemma 3}

Let a Hückel Hamiltonian be that of an alternant system with an equal number of starred and unstarred atomic orbitals. Assume furthermore that there are no nonbonding molecular orbitals. Let us call the starred orbitals $1,3,5, \ldots . N-1$ and the unstarred orbitals $2,4,6, \ldots N$. Consider only the $S_{z}$ $=0$ manifold localized SDs [i.e., the SDs such as those shown in Fig. 1(b) (iii) for trimethylenemethane]. The SD which provides the largest contribution to $\Psi_{\text {Hückel }}$ is in general $|2,4,6, \ldots . ., \overline{1}, \overline{3}, \ldots \overline{N-1}|$.

\section{Proof}

To simplify notation we give the proof for $N=6$. In this case

$$
\Psi_{\text {Hückel }}=\left|\phi_{1} \phi_{2} \phi_{3} \bar{\phi}_{1} \bar{\phi}_{2} \bar{\phi}_{3}\right|
$$

and

$$
\begin{aligned}
& \left\langle|135 \overline{2} \overline{4} \overline{6}| \mid \Psi_{\text {Hückel }}\right\rangle \\
& \quad=\left|\begin{array}{lll}
\phi_{11} & \phi_{12} & \phi_{13} \\
\phi_{31} & \phi_{32} & \phi_{33} \\
\phi_{51} & \phi_{52} & \phi_{53}
\end{array}\right| \cdot\left|\begin{array}{lll}
\phi_{21} & \phi_{22} & \phi_{23} \\
\phi_{41} & \phi_{42} & \phi_{43} \\
\phi_{61} & \phi_{62} & \phi_{63}
\end{array}\right| .
\end{aligned}
$$

Recall that in alternant systems, eigenfunctions have the property that if

$$
\left(\phi_{1 a}, \phi_{3 a}, \phi_{5 a}, \phi_{2 a}, \phi_{4 a}, \phi_{6 a}\right) \text { and }\left(\phi_{1 b}, \phi_{3 b}, \phi_{5 b}, \phi_{2 b}, \phi_{4 b}, \phi_{6 b}\right)
$$

are eigenvectors then so are

$$
\left(\phi_{1 a}, \phi_{3 a}, \phi_{5 a},-\phi_{2 a},-\phi_{4 a},-\phi_{6 a}\right)
$$

$$
\left(\phi_{1 b}, \phi_{3 b}, \phi_{5 b},-\phi_{2 b},-\phi_{4 b},-\phi_{6 b}\right) \text {. }
$$

Due to the orthonormality of the above vectors we see that

$$
\sum_{k=1}^{3} \phi_{2 k, a} \phi_{2 k, b} \pm \sum_{k=1}^{3} \phi_{2 k-1, a} \phi_{2 k-1, b}=0 \text {. }
$$

Therefore,

$\sum \phi_{2 k, a} \phi_{2 k, b}=\sum \phi_{2 k-1, a} \phi_{2 k-1, b}=0$.

Thus the two determinants shown in Eq. (12) are both composed of orthogonal 3-vectors. Recalling that determinants are volumes, we have in some sense maximized the volume of each determinant. Were we to have picked a different SD, we would in general not have orthogonal vectors and hence we would find a smaller coefficient for such an SD. Q.E.D.

For the next lemma we adopt the following phase convention: the spatial orbital position remains fixed in localized SDs. Thus for trimethylenemethane the six $S_{z}=0$ manifold localized SDs are $|12 \overline{3} \overline{4}|,|\overline{1} 234|,|\overline{1} 2 \overline{3} 4|,|1 \overline{2} 3 \overline{4}|$, $|1 \overline{2} \overline{3} 4|$, and $|\overline{1} 23 \overline{4}|$.

\section{Lemma 4}

Consider an alternant system with an equal number of starred and unstarred atomic orbitals. Consider the starred and unstarred sets of the HD Hamiltonians SDs. It is well known that $\Psi_{\text {HD }}$ has positive contributions from all its starred SDs and negative contributions from all its unstarred SDs. ${ }^{5}$ This is also true, in general, for the same localized SDs in $\Psi_{\text {Hückel }}$.

\section{Proof}

We will consider the example used in the proof of Lemma 3. We begin by comparing the relative phases of

$$
\left\langle|135 \overline{2} \overline{4} \overline{6}| \mid \Psi_{\text {Hückel }}\right\rangle \text { and }\left\langle|136 \overline{2} \overline{45}| \mid \Psi_{\text {Hückel }}\right\rangle .
$$

Recalling Eq. (12) and noting,

$\left\langle|136 \overline{2} \overline{4} \overline{5}| \mid \Psi_{\text {Hückel }}\right\rangle$

$$
=\left|\begin{array}{lll}
\phi_{11} & \phi_{12} & \phi_{13} \\
\phi_{31} & \phi_{32} & \phi_{33} \\
\phi_{61} & \phi_{62} & \phi_{63}
\end{array}\right| \cdot\left|\begin{array}{lll}
\phi_{21} & \phi_{22} & \phi_{23} \\
\phi_{41} & \phi_{42} & \phi_{43} \\
\phi_{51} & \phi_{52} & \phi_{53}
\end{array}\right|
$$

we find,

$$
\begin{aligned}
\left\langle|136 \overline{2} \overline{4} \overline{5}| \mid \Psi_{\text {Hückel }}\right\rangle= & \left\{\left(\phi_{61}, \phi_{62}, \phi_{63}\right) \cdot\left(\phi_{51}, \phi_{52}, \phi_{53}\right)\right\}^{2} \\
& \times\left\langle|135 \overline{2} \overline{4} \overline{6}| \mid \Psi_{\text {Hückel }}\right\rangle .
\end{aligned}
$$

Furthermore,

$$
\left\langle|13 \overline{5} \overline{2} \overline{4} 6| \mid \Psi_{\text {Hückel }}\right\rangle=-\left\langle|136 \overline{245}| \mid \Psi_{\text {Hückel }}\right\rangle .
$$

This is just the result we want to prove. In general though, one does not have only orthogonal vectors. In general one must compare

$$
A=\left|\begin{array}{lll}
x_{11} & x_{12} & x_{13} \\
x_{21} & x_{22} & x_{23} \\
x_{31} & x_{32} & x_{33}
\end{array}\right| \cdot\left|\begin{array}{lll}
y_{11} & y_{12} & y_{13} \\
y_{21} & y_{22} & y_{23} \\
y_{31} & y_{32} & y_{33}
\end{array}\right|
$$

with 
TABLE II. Comparison of $\Psi_{\text {Hückel }}$ and $\Psi_{\text {HD }}$ for some six atom systems.

\begin{tabular}{|c|c|c|c|c|c|c|c|c|c|c|}
\hline $\begin{array}{l}\text { Down spin } \\
\text { atoms }\end{array}$ & $2 \Psi_{\text {Hückel }}$ & $2 \Psi_{\mathrm{HD}}$ & $3 \Psi_{\text {Hückel }}$ & $3 \Psi_{\mathrm{HD}}$ & $4 \Psi_{\text {Hückel }}$ & $4 \Psi_{\mathrm{HD}}$ & $\begin{array}{c}4 \Psi_{\text {HD plus }} \\
4 \text { th order } \\
\text { corrections }^{\mathrm{c}}\end{array}$ & $5 \Psi_{\text {Huickel }}$ & $5 \Psi_{\mathrm{HD}}$ & $\begin{array}{c}5 \Psi_{\text {HO }} \text { plus } \\
\text { 4th order } \\
\text { corrections }^{d}\end{array}$ \\
\hline $4,5,6$ & -0.112 & -0.131 & -0.007 & -0.021 & -0.008 & -0.051 & -0.004 & -0.759 & -0.517 & -0.700 \\
\hline $3,5,6$ & 0.444 & 0.434 & 0.091 & 0.135 & 0.166 & 0.225 & 0.183 & 0.234 & 0.183 & 0.285 \\
\hline $3,4,6$ & -0.444 & -0.434 & -0.234 & -0.293 & -0.166 & -0.225 & -0.183 & -0.091 & -0.205 & -0.114 \\
\hline $3,4,5$ & 0.112 & 0.131 & 0.150 & 0.178 & 0.008 & 0.051 & 0.004 & 0.616 & 0.538 & 0.529 \\
\hline $2,5,6$ & -0.444 & -0.434 & -0.234 & -0.293 & -0.825 & -0.725 & -0.820 & -0.234 & -0.183 & -0.285 \\
\hline $2,4,6^{c}$ & 1.000 & 1.000 & 1.000 & 1.000 & 1.000 & 1.000 & 1.000 & 1.000 & 1.000 & 1.000 \\
\hline $2,4,5$ & -0.444 & -0.434 & -0.759 & -0.686 & -0.166 & -0.225 & -0.183 & -0.007 & -0.300 & -0.014 \\
\hline $2,3,6$ & -0.444 & -0.434 & -0.616 & -0.529 & -0.166 & -0.225 & -0.183 & -0.150 & -0.278 & -0.186 \\
\hline $2,3,5$ & 0.444 & 0.434 & 0.759 & 0.686 & 0.825 & 0.725 & 0.820 & 0.150 & 0.278 & 0.186 \\
\hline $2,3,4$ & -0.112 & -0.131 & -0.150 & -0.178 & -0.667 & -0.550 & -0.634 & -0.759 & -0.517 & -0.700 \\
\hline
\end{tabular}

a SD phase convention is such that first and second row are, respectively, $|123 \overline{456}|$ and $|12 \overline{3} 4 \overline{5} \overline{6}|$.

${ }^{b}$ Each column presents $\Psi$ in column vector form.

${ }^{\circ}(U / \beta)^{2}=0.10$.

$d(U / B)^{2}=0.03$.

${ }^{e}$ Vectors are normalized so that $\langle|1 \overline{2} 3 \overline{4} 5 \overline{6}| \Psi\rangle=1.00$.

$$
B=\left|\begin{array}{lll}
x_{11} & x_{12} & x_{13} \\
x_{21} & x_{22} & x_{23} \\
y_{31} & y_{32} & y_{33}
\end{array}\right| \cdot\left|\begin{array}{lll}
y_{11} & y_{12} & y_{13} \\
y_{21} & y_{22} & y_{23} \\
x_{31} & x_{32} & x_{33}
\end{array}\right| .
$$

We see that,

$$
B=A\left(\mathbf{y}_{3}^{\prime} \cdot \chi_{3}\right)\left(\chi_{3}^{\prime} \cdot \mathbf{y}_{3}\right),
$$

$$
\mathbf{y}_{i}=\left(\mathrm{y}_{i 1}, \mathrm{y}_{i 2}, \mathrm{y}_{i 3}\right) \text { and } \chi_{i}=\left(\chi_{i 1}, \chi_{i 2}, \chi_{i 3}\right)
$$

and $y_{3}^{\prime}$ is the dual of $y_{3}$, i.e., that portion of $y_{3}$ which is orthogonal to $y_{1}$ and $y_{2}$ and similarly for $\chi_{3}^{1}$.

Hence in general $B$ and $A$ are not of the same phase. But $B$ and $A$ will be of different signs only when $y_{3}^{\prime}$ and $y_{3}$ and/or $\chi_{3}^{\prime}$ and $\chi_{3}$ differ significantly from each other. If this is the case, then $A$ or $B$ must be rather small. Hence our rule will in general break down only for small coefficients. Q.E.D.

\section{Numerical illustrations}

It is useful to examine some numerical examples of these lemmas. In Table II we consider four six-orbital systems, 25.<smiles>o1oooo1</smiles><smiles>[O-]O[Os]</smiles><smiles>[O-]O[Ga]O[Na]</smiles>

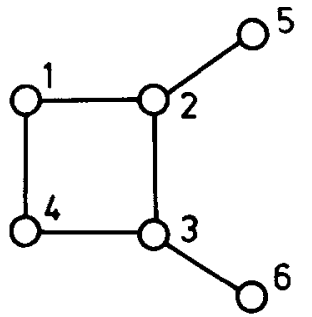

They may be viewed, for example, as representations of the $\pi$ orbitals of unsaturated hydrocarbons. 2 would then represent benzene, 3 hexatriene, and so forth. Table II shows there is a strong correlation in the contribution of the localized SDs to $\Psi_{\mathrm{HD}}$ and to $\Psi_{\text {Huckel }}$. In particular we note:

(a) The relative size of each SD in $\Psi_{\mathrm{HD}}$ and $\Psi_{\text {Hückel }}$ depends on its coordination number. Recalling that the coordination number of Hückel and HD diagrams are proportional to each other, we find (as predicted by Lemma 2) that the size of a given SDs contribution to either $\Psi_{\mathrm{HD}}$ or $\Psi_{\text {Hückel }}$ is in rough accord. The only exception to this rule is found in the $|1 \overline{2} 3 \overline{4} \overline{5} 6|$ SD for 5 .

(b) As predicted by Lemma 3, the largest term is invariably $|1 \overline{2} 3 \overline{4} 5 \overline{6}|$.

(c) As predicted by Lemma 4, the relative phase is the same for each SD coefficient.

We see that the agreement is best for $\mathbf{2}$ and $\mathbf{3}$ and worst for 4 and 5 . We shall show in a later section that this increased disparity is due to the presence of a square in 5 , and to the nonhomogeneity of both 4 and 5 .

This latter concept of homogeneity will be elaborated later. We anticipate the results of a following section by also including in Table II the fourth order corrections to $\Psi_{\mathrm{HD}}$. It can be seen that these corrections substantially improve the agreement between the spin Hamiltonian and the Hückel Hamiltonian.

Table III treats in an identical manner three 8-orbital systems, 6-8.

We observe the same trends as in Table II and again we observe that the least homogeneous system, 8 , needs fourth 


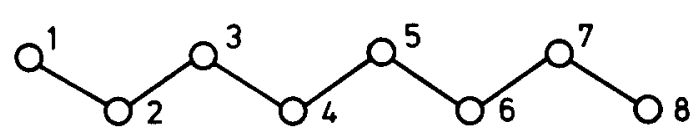

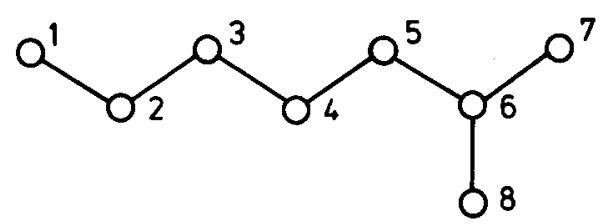

order corrections to its $\Psi_{\mathrm{HD}} \cdot 8$ also illustrates the approximate nature of Lemmas 3 and 4 . We find for example that the largest contributor to $\Psi_{\text {Hïckel }}$ is not $|1 \overline{2} 3 \overline{4} 5 \overline{6} \overline{7} \overline{8}|$ but rather $|1 \overline{2} 34 \overline{5} 6 \overline{7} \overline{8}|$, not in accord with Lemma 3 . Similarly Lemma 4 breaks down over several of the smaller terms in $\Psi_{\text {Hückel }}$, e.g., $|123 \overline{4} 5 \overline{6} \overline{7} \overline{8}|$.

\section{Hubbard Hamiltonian}

In the preceding section we have illustrated in a somewhat formal manner that the Hückel and HD ground states in alternant systems have a similar localized electronic configuration. Why is this true and what does this mean chemically? One way of viewing our preceding results is as follows:

The Hückel and the Heisenberg Dirac Hamiltonians account for two different interactions. The Hückel Hamiltonian concerns itself only with one electron terms while the HD Hamiltonian considers electron correlation energies. In many chemical systems, neither Hamiltonian is really adequate as often the two electron terms and the one electron terms are of the same order of magnitude. But if the Hückel ground state and the HD ground state are qualitatively similar in their localized part of the wave function then one may infer that the region of chemical interest will also show simi-

TABLE III. Comparison of $\Psi_{\text {Hückel }}$ and $\Psi_{H D}$ for eight atom systems.

\begin{tabular}{|c|c|c|c|c|c|c|c|}
\hline $\begin{array}{l}\text { Down spin } \\
\text { atoms }^{\mathrm{a}}\end{array}$ & $6 \Psi_{\text {Hückel }}^{b}$ & $6 \Psi_{\mathrm{HD}}$ & $7 \Psi_{\text {Huickel }}$ & $7 \Psi_{\mathrm{HD}}$ & $8 \Psi_{\text {Hückel }}$ & $8 \Psi_{\mathrm{HD}}$ & $\begin{array}{l}8 \Psi_{\text {HD }} \\
\text { fourth } \\
\text { order }^{c}\end{array}$ \\
\hline $5,6,7,8$ & 0.001 & 0.000 & -0.001 & 0.001 & 0.006 & 0.011 & -0.015 \\
\hline $4,6,7,8$ & -0.001 & -0.004 & 0.002 & -0.010 & 0.023 & -0.044 & 0.056 \\
\hline $4,5,7,8$ & 0.000 & 0.016 & 0.041 & 0.020 & -0.021 & 0.042 & -0.051 \\
\hline $4,5,6,8$ & -0.013 & -0.026 & -0.038 & -0.016 & -0.008 & -0.012 & 0.008 \\
\hline $4,5,6,7$ & 0.013 & 0.014 & -0.003 & 0.004 & 0.001 & 0.003 & 0.003 \\
\hline $3,6,7,8$ & 0.000 & 0.016 & 0.001 & 0.034 & 0.081 & 0.168 & 0.118 \\
\hline $3,5,7,8$ & -0.030 & -0.084 & -0.112 & -0.119 & -0.087 & -0.220 & -0.149 \\
\hline $3,5,6,8$ & 0.127 & 0.157 & 0.113 & 0.108 & 0.011 & 0.065 & 0.022 \\
\hline $3,5,6,7$ & -0.098 & -0.089 & -0.001 & -0.024 & -0.011 & -0.023 & 0.023 \\
\hline $3,4,7,8$ & 0.030 & 0.094 & 0.183 & 0.210 & 0.004 & 0.054 & 0.026 \\
\hline $3,4,6,8$ & -0.185 & -0.300 & -0.197 & -0.292 & -0.091 & -0.226 & -0.138 \\
\hline $3,4,6,7$ & 0.156 & 0.194 & 0.011 & 0.057 & -0.017 & 0.048 & -0.062 \\
\hline $3,4,5,8$ & 0.070 & 0.131 & 0.009 & 0.063 & 0.082 & 0.162 & 0.122 \\
\hline $3,4,5,7$ & -0.070 & -0.158 & -0.120 & -0.174 & 0.022 & -0.038 & 0.051 \\
\hline $3,4,5,6$ & 0.000 & 0.038 & 0.114 & 0.137 & 0.006 & 0.011 & -0.015 \\
\hline $2,6,7,8$ & -0.013 & -0.026 & 0.005 & -0.060 & -0.908 & -0.544 & -0.796 \\
\hline $2,5,7,8$ & 0.127 & 0.157 & 0.126 & 0.248 & 1.011 & 0.711 & 1.011 \\
\hline $2,5,6,8$ & -0.351 & -0.323 & -0.141 & -0.243 & -0.091 & -0.226 & -0.138 \\
\hline $2,5,6,7$ & 0.236 & 0.192 & 0.010 & 0.053 & -0.017 & 0.048 & -0.062 \\
\hline $2,4,7,8$ & -0.185 & -0.300 & -0.927 & -0.720 & -0.087 & -0.220 & -0.149 \\
\hline $2,4,6,8^{d}$ & 1.000 & 1.000 & 1.000 & 1.000 & 1.000 & 1.000 & 1.000 \\
\hline $2,4,6,7$ & -0.802 & -0.670 & -0.080 & -0.211 & -0.028 & -0.192 & -0.111 \\
\hline $2,4,5,8$ & -0.566 & -0.493 & -0.068 & -0.222 & -0.923 & -0.718 & -0.927 \\
\hline $2,4,5,7$ & 0.623 & 0.622 & 0.827 & 0.674 & 0.021 & 0.185 & 0.115 \\
\hline $2,4,5,6$ & -0.070 & -0.158 & -0.752 & -0.520 & 0.023 & -0.044 & 0.056 \\
\hline $2,3,7,8$ & 0.070 & 0.131 & 0.682 & 0.394 & -0.022 & 0.042 & -0.051 \\
\hline $2,3,6,8$ & -0.566 & -0.493 & -0.744 & -0.524 & -0.023 & -0.191 & -0.117 \\
\hline $2,3,6,7$ & 0.508 & 0.372 & 0.056 & 0.155 & 0.871 & 0.525 & 0.846 \\
\hline $2,3,5,8$ & 0.634 & 0.465 & 0.127 & 0.160 & 0.021 & 0.185 & 0.115 \\
\hline $2,3,5,7$ & -0.802 & -0.670 & -0.766 & -0.683 & -0.924 & -0.717 & -0.926 \\
\hline $2,3,5,6$ & 0.156 & 0.195 & 0.700 & 0.499 & 0.081 & 0.168 & 0.118 \\
\hline $2,3,4,8$ & -0.151 & -0.117 & -0.005 & -0.034 & 0.022 & -0.038 & 0.051 \\
\hline $2,3,4,7$ & 0.236 & 0.192 & 0.067 & 0.149 & 0.082 & 0.162 & 0.122 \\
\hline $2,3,4,6$ & -0.098 & -0.089 & -0.054 & -0.150 & -0.908 & -0.544 & -0.796 \\
\hline $2,3,4,5$ & 0.013 & 0.014 & -0.007 & 0.034 & 0.798 & 0.409 & 0.637 \\
\hline
\end{tabular}

SD phase convention is such that the first and second rows are, for example, $|1234 \overline{5} \overline{6} \overline{8}|$ and $|123 \overline{4} 5 \overline{67 \overline{8}}|$.

${ }^{b}$ Each column presents the $\Psi$ in question in vector form.

c $(U / \beta)^{2}=0.135$.

dVectors are normalized so that this coefficient equals 1.00 . 
TABLE IV. $\Psi_{\text {Hubbard }}$ for 9 .

\begin{tabular}{lcccccc}
\hline \hline & $U=0$ & $U=1$ & $U=2$ & $U=4$ & $U=10$ & $U=50$ \\
\hline$\left\langle|12 \overline{3} \overline{4}| \Psi_{\text {Hubbard }}\right\rangle^{\mathrm{a}}$ & 0.20 & 0.22 & 0.24 & 0.26 & 0.27 & 0.27 \\
$\left\langle|1 \overline{2} 3 \overline{4}| \Psi_{\text {Hubburd }}\right\rangle^{\mathrm{a}}$ & 1.00 & 1.00 & 1.00 & 1.00 & 1.00 & 1.00 \\
$\left\langle|1 \overline{2} \overline{3} 4| \Psi_{\text {Hubbard }}\right\rangle^{\mathrm{a}}$ & 0.80 & 0.78 & 0.76 & 0.75 & 0.74 & 0.73 \\
$\left\langle\left.\left\langle P \Psi_{\text {Hubbard }} \mid \Psi_{\text {Hubbard }}\right\rangle\right|^{2}\right.$ & $21.0 \%$ & $32.8 \%$ & $46.0 \%$ & $67.8 \%$ & $91.6 \%$ & $99.6 \%$ \\
\hline \hline
\end{tabular}

${ }^{a}$ Largest coefficient normalized to 1.00 .

lar qualitative behavior. ${ }^{6}$ It is therefore of interest to place both Hückel and HD Hamiltonians within the context of a Hamiltonian which considers both one and two electron terms. As we have mentioned earlier, this corresponds to the Hubbard Hamiltonian.

We now consider the Hubbard Hamiltonian of butadiene, 9. In Table IV we show how the localized SDs slowly evolve from the Hückel antipode where $U / \beta=0$ to the HD<smiles>[O]O[O]</smiles>

antipode where $U \gg|\beta|$. As may be seen, the change is slow, continuous and slight. The entire effect of the changing $U / \beta$ parameter is spent on changing the percent contribution of this localized configuration from the HD antipode to the Hückel antipode. This is also shown in Table IV.

One particularly useful way of viewing the Hubbard Hamiltonian is to tabulate the molecular orbital provenance of the Hubbard ground state. To do so, one uses as a basis set of the Hubbard space SDs composed of molecular orbitals. Thus in the case of the four atom chain 9, one has four molecular orbitals, $\phi_{1}, \phi_{2}, \phi_{3}$, and $\phi_{4}$. We therefore express the Hubbard ground state as

$$
\Psi_{\text {Hubbard }}=\sum_{a b c d} \alpha_{a b c d}\left|\phi_{a} \phi_{b} \bar{\phi}_{c} \bar{\phi}_{d}\right|,
$$

where $a \neq b$ and $c \neq d$.

We then arbitrarily assign a quarter of $\left|\alpha_{a b c d}\right|^{2}$ to, re-

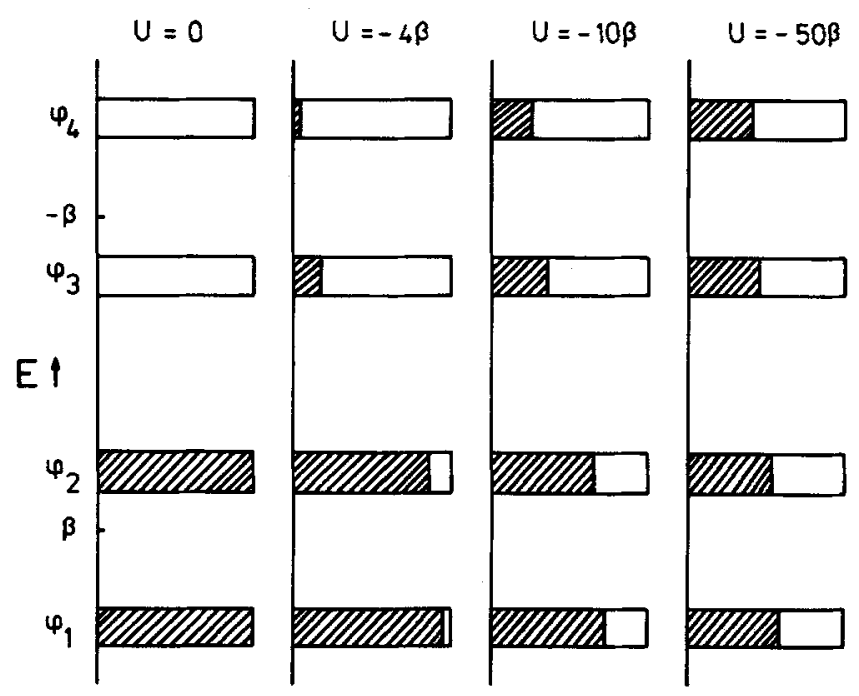

FIG. 2. Molecular orbital occupation as a function of $|U / \beta|$ for 9 . Going from left to right we consider increasingly large $|U / \beta|$. Striped area represents filled orbitals, nonstriped area unfilled ones. Note even at $U=-10 \beta$ the bonding orbitals are substantially more occupied. spectively, $\phi_{a}, \phi_{b}, \phi_{c}$, and $\phi_{d}$. In this way one can represent the molecular orbital provenance of $\Psi_{\text {Hubbard }}$.

We plot the results of such a fractionation in Fig. 2. It may be seen that the switching on of the $U$ parameter is reminiscent to the effect of temperature. The bonding orbitals empty out and the antibonding orbitals fill up as $U$ becomes increasingly dominant. This is an intuitive result. In the limit of very large $U$, molecular orbital stabilization energy becomes immaterial. The only energetic requirement in the limit of high $U$ is that electrons should be as localized as possible. Total localization corresponds to a half-filling of every molecular orbital.

\section{Fourth order correction}

As we have seen in the previous sections, the agreement between the localized portion of the Huckel and HD ground states is not always excellent. As we show in this section, this divergence comes from changes which occur in the region where $|U / \beta|>1$. We prove this result via perturbation theory. Before showing how this is done in practice, we would like to note, that there is no mathematical reason why the discrepencies have to be eliminated by higher order correction. A perturbation expansion starting from either the Hückel or Heisenberg Dirac antipode breaks down at $U=|\beta|$. The reason why the expansion method works is because the changeover from Hückel to Heisenberg antipodes is of a continuous type for the alternant systems.

In order to calculate higher order terms in the HD Hamiltonian we need to recall several results from effective Hamiltonian perturbation theory. ${ }^{7}$ In Table $\mathrm{V}$ we list the various

TABLE V. Rayleigh-Schrödinger perturbation formulas.

\begin{tabular}{cl}
\hline \hline $\begin{array}{c}\text { Order of } \\
\text { perturbation }\end{array}$ & Expression \\
\hline $1^{\mathrm{a}}$ & $P V P$ \\
$2^{\mathrm{b}}$ & $P V \frac{Q}{\Delta E} V P$ \\
3 & $P V \frac{Q}{\Delta E} V \frac{Q}{\Delta E} V P-P V \frac{Q}{(\Delta E)^{2}} V P V P$ \\
4 & $P V \frac{Q}{\Delta E} V \frac{Q}{\Delta E} V \frac{Q}{\Delta E} V P-P V \frac{Q}{\Delta E} V \frac{Q}{(\Delta E)^{2}} V P V P$ \\
& $-P V \frac{Q}{(\Delta E)^{2}} V \frac{Q}{\Delta E} V P V P-P V \frac{Q}{(\Delta E)^{2}} V P V \frac{Q}{\Delta E} V P$ \\
& $+P \frac{2 Q}{(\Delta E)^{3}} V P V P V P$ \\
\hline \hline
\end{tabular}

" $V$ is the perturbing Hamiltonian elements, i.e., $H=H_{0}+V$, where $H$ is the full Hamiltonian and $H_{0}$ the unperturbed Hamiltonian. $P$ is the projection operator onto the effective space.

${ }^{\mathrm{b}} Q=1-P . \Delta E=E_{0}-H_{0}$, where $E_{0}$ equals the unperturbed diagonal element of the degenerate vectors which compose the effective space. 
order corrections to the Hamiltonian. In our case $P V P=0$, and furthermore for alternant systems there are no third order terms.

Some computation shows the fourth order correction in the absence of squares is

$\mathbf{H}_{4 a}=\sum_{i>j}-\frac{\beta^{4}}{U^{3}}\left\{b_{i j}\left(16 \mathbf{S}_{i} \cdot \mathbf{S}_{j}\right)-C_{i j}\left(4 \mathbf{S}_{i} \cdot \mathbf{S}_{j}\right)-4 b_{i j}+c_{i j}\right\}$,

where

$$
b_{i j}=\left\{\begin{array}{ll}
1 & \text { if } \beta_{i j}=\beta \\
0 & \text { otherwise }
\end{array},\right.
$$

and $c_{i j}$ is the number of first nearest neighbors which both $\chi_{i}$ and $\chi_{j}$ share in common.

When four member rings are present one has the additional correction

$$
\begin{aligned}
\mathbf{H}_{4 b}= & \frac{\beta^{4}}{U^{3}} \sum_{i j, k, l} d_{i j k l}\left\{1 0 \left[\left(\mathbf{S}_{i} \cdot \mathbf{S}_{j}\right)\left(\mathbf{S}_{k} \cdot \mathbf{S}_{l}\right)+\left(\mathbf{S}_{i} \cdot \mathbf{S}_{l}\right)\left(\mathbf{S}_{j} \cdot \mathbf{S}_{k}\right)\right.\right. \\
& \left.-\left(\mathbf{S}_{i} \cdot \mathbf{S}_{k}\right)\left(\mathbf{S}_{j} \cdot \mathbf{S}_{l}\right)\right]-\frac{1}{2}\left[\mathbf{S}_{i} \cdot \mathbf{S}_{j}+\mathbf{S}_{j} \cdot \mathbf{S}_{l}+\mathbf{S}_{i} \cdot \mathbf{S}_{l}\right. \\
& \left.\left.+\mathbf{S}_{i} \cdot \mathbf{S}_{k}+\mathbf{S}_{j} \cdot \mathbf{S}_{k}+\mathbf{S}_{l} \cdot \mathbf{S}_{k}\right]+\frac{1}{8}\right\},
\end{aligned}
$$

where

$$
d_{i j k l}= \begin{cases}1 & \text { if } b_{i j} b_{j k} b_{k l} b_{i l}=1 \\ 0 & \text { otherwise }\end{cases}
$$

The total fourth order correction is, therefore,

$$
\mathbf{H}_{4}=\mathbf{H}_{4 a}+\mathbf{H}_{4 b} \text {. }
$$

In the case of cyclobutadiene 10 we find

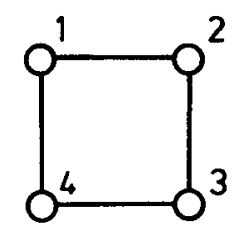

$$
\begin{aligned}
\mathbf{H}_{4}= & \frac{\beta^{4}}{U^{3}}\left\{80\left(\mathbf{S}_{1} \cdot \mathbf{S}_{2}\right)\left(\mathbf{S}_{3} \cdot \mathbf{S}_{4}\right)+80\left(\mathbf{S}_{1} \cdot \mathbf{S}_{4}\right)\left(\mathbf{S}_{2} \cdot \mathbf{S}_{3}\right)\right. \\
& -80\left(\mathbf{S}_{1} \cdot \mathbf{S}_{3}\right)\left(\mathbf{S}_{2} \cdot \mathbf{S}_{4}\right)-4 \mathbf{S}_{1} \cdot \mathbf{S}_{3}-4 \mathbf{S}_{2} \cdot \mathbf{S}_{4} \\
& \left.+20\left[\mathbf{S}_{1} \cdot \mathbf{S}_{2}+\mathbf{S}_{2} \cdot \mathbf{S}_{3}+\mathbf{S}_{3} \cdot \mathbf{S}_{4}+\mathbf{S}_{1} \cdot \mathbf{S}_{4}\right]-13\right\} .
\end{aligned}
$$

We can see there are three types of terms which appear in the fourth order:

(a) A ferromagnetic interaction between nearest neighbors. In the absence of four member rings this effect is exactly proportional to the HD Hamiltonian. By itself therefore, it introduces no change in the functional form of the Hamiltonian. Except in the presence of squares this force is of little qualitative importance.

(b) An antiferromagnetic (AF) interaction between second nearest neighbors. We illustrate this effect for trimethylenemethane, in Fig. 3. We may understand its effect in the following manner: in general we will be placing an AF force between two SDs which are in ferromagnetic alignment to one another. If the second order solution has endowed the two SDs with disparate coefficients this AF force will tend to further increase the coefficient of the larger SD and further decrease the coefficient of the smaller one. In the

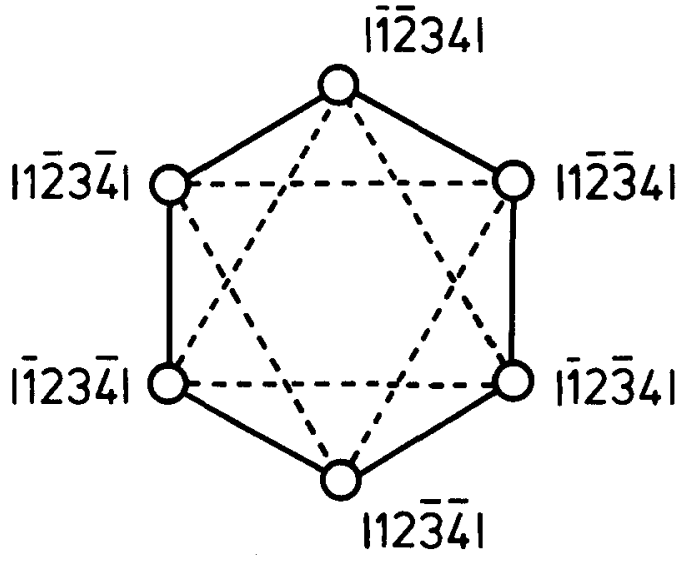

FIG. 3. Fourth order correction to the HD Hamiltonian of 1. Solid lines are second order terms, dotted lines are fourth order terms. All lines are of AF type. Compare to Fig. 1(b) (iii).

case of Fig. 3, though, all the second nearest neighbor interactions are between SDs which are of identical magnitude. Hence this force is of little importance. Thus when the system exhibits more homogeneity, of the kind found in 1, this force also is of lesser importance.

(c) A four body interaction. Evaluation of Eq. (25) shows that the four body term is of the type shown in 11. This force is quite important.

Inclusion of this force genuinely improves the similarity between $P \Psi_{\text {Hückel }}$ and $P \Psi_{\mathrm{HD}}$.

We give numerical illustrations of this improvement in Table VI for 12. Other examples are given in Tables II and III.
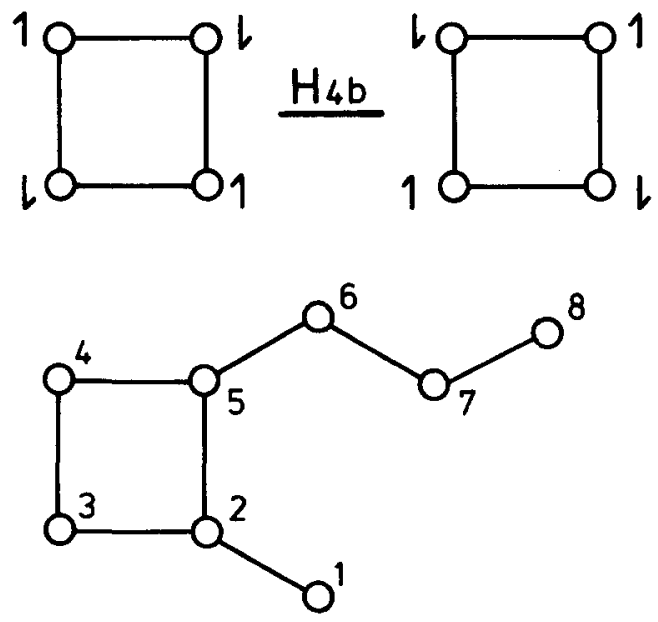

12

\section{Nonalternant systems}

The results presented in the earlier sections are applicable only to alternant systems. Nonalternant systems present a radically different behavior. We show this by examining 13. 


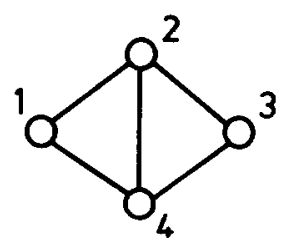

13

In Table VII we present in an analogous fashion to Table IV the results of a Hubbard calculation for 13. It may be seen that the system undergoes a delocalized-electron to localized-electron transition. This transition occurs at $U=-2.7951 \beta$. At this $U / \beta$ ratio there is a crossover between the ground state and the first excited state of the Hubbard Hamiltonian. The two states correspond to different irreducible symmetry representations of the Hubbard Hamiltonian and hence the crossing is a true one, not an avoided one. This crossing leads to unanalytic behavior between the Hückel and HD antipodes. We illustrate this in Fig. 4. In this figure we contrast butadiene, 9 to 13 .

The reason for the crossover in $\mathbf{1 3}$ is also of interest. Let

TABLE VI. $\Psi_{\text {Hücket }}$ and $\Psi_{\mathrm{HD}}$ for 12.

\begin{tabular}{|c|c|c|c|}
\hline $\begin{array}{l}\text { Down spin } \\
\text { atoms }^{\mathrm{a}}\end{array}$ & $12 \Psi_{\text {Hückel }}{ }^{b}$ & $\begin{array}{l}12 \Psi_{\mathrm{HD}} \text { with some fourth } \\
\text { order correction }^{\mathrm{c}}\end{array}$ & $12 \Psi_{\mathrm{HD}}$ \\
\hline $5,6,7,8$ & 0.001 & 0.002 & 0.005 \\
\hline $4,6,7,8$ & -0.014 & -0.006 & -0.016 \\
\hline $4,5,7,8$ & 0.029 & 0.049 & 0.101 \\
\hline $4,5,6,8$ & -0.026 & -0.121 & -0.218 \\
\hline $4,5,6,7$ & 0.010 & 0.075 & 0.129 \\
\hline $3,6,7,8$ & 0.004 & 0.004 & 0.026 \\
\hline $3,5,7,8$ & -0.004 & -0.014 & -0.166 \\
\hline $3,5,6,8$ & -0.002 & 0.017 & 0.326 \\
\hline $3,5,6,7$ & 0.003 & -0.010 & -0.190 \\
\hline $3,4,7,8$ & 0.008 & 0.048 & 0.094 \\
\hline $3,4,6,8$ & -0.081 & -0.179 & -0.282 \\
\hline $3,4,6,7$ & 0.083 & 0.133 & 0.178 \\
\hline $3,4,5,8$ & 0.086 & 0.199 & 0.131 \\
\hline $3,4,5,7$ & -0.119 & -0.282 & -0.161 \\
\hline $3,4,5,6$ & 0.024 & 0.084 & 0.044 \\
\hline $2,6,7,8$ & -0.003 & -0.016 & -0.027 \\
\hline $2,5,7,8$ & 0.130 & 0.097 & 0.147 \\
\hline $2,5,6,8$ & -0.218 & -0.207 & -0.299 \\
\hline $2,5,6,7$ & 0.134 & 0.123 & 0.175 \\
\hline $2,4,7,8$ & -0.221 & -0.290 & -0.319 \\
\hline $2,4,6,8^{d}$ & 1.000 & 1.000 & 1.000 \\
\hline $2,4,6,7$ & -0.762 & -0.688 & -0.638 \\
\hline $2,4,5,8$ & -0.634 & -0.509 & -0.372 \\
\hline $2,4,5,7$ & 0.740 & 0.653 & 0.444 \\
\hline $2,4,5,6$ & -0.122 & -0.163 & -0.110 \\
\hline $2,3,7,8$ & 0.115 & 0.125 & 0.156 \\
\hline $2,3,6,8$ & -0.659 & -0.495 & -0.513 \\
\hline $2,3,6,7$ & 0.544 & 0.382 & 0.359 \\
\hline $2,3,5,8$ & 0.682 & 0.485 & 0.347 \\
\hline $2,3,5,7$ & -0.879 & -0.694 & -0.483 \\
\hline $2,3,5,6$ & 0.198 & 0.199 & 0.140 \\
\hline $2,3,4,8$ & -0.147 & -0.191 & -0.118 \\
\hline $2,3,4,7$ & 0.244 & 0.308 & 0.187 \\
\hline $2,3,4,6$ & -0.112 & -0.135 & -0.087 \\
\hline $2,3,4,5$ & 0.013 & 0.015 & 0.012 \\
\hline
\end{tabular}

"SD phase convention is such that first and second rows are, respectively, $|1234 \overline{5} \overline{6} \overline{8}|$ and $|123 \overline{4} 5 \overline{678}|$.

'Each column presents the $\Psi$ in vector form.

${ }^{c}(U / \beta)^{2}=0.3$

dVectors are normalized so that this coefficient equals 1.00.
TABLE VII. $\Psi_{\text {Huthard }}$ for 13.

\begin{tabular}{lccccc}
\hline \hline & $U=0$ & $U=2$ & $U=4$ & $U=10$ & $U=50$ \\
\hline$\left\langle|12 \overline{3} \overline{4}| \Psi_{\text {Hubbard }}\right\rangle^{\mathrm{a}}$ & 1.00 & 1.00 & 0.50 & 0.50 & 0.50 \\
$\left\langle|1 \overline{2} 3 \overline{4}| \Psi_{\text {Hubbard }}\right\rangle^{\mathrm{a}}$ & 0.00 & 0.00 & 1.00 & 1.00 & 1.00 \\
$|\langle P \Psi \mid \Psi\rangle|^{2}$ & $9.6 \%$ & $29.2 \%$ & $71.0 \%$ & $90.5 \%$ & $99.5 \%$ \\
\hline
\end{tabular}

"Largest coefficient normalized to 1.00 .

us first examine the $\Psi_{\mathrm{HD}}$ antipode. The SDs $|1 \overline{2} 3 \overline{4}|$ and $|\overline{1} 2 \overline{3} 4|$ contain only one pair of like spins on bonded atoms while the other four SDs contain two such energetically unfavorable pairs. It is to be expected that $|1 \overline{2} 3 \overline{4}|$ and $|\overline{1} 2 \overline{3} 4|$ both contribute to $\Psi_{\text {HD }}$. Furthermore 13 is of $D_{2 h}$ symmetry. The permutational transposition (13) corresponds to two of the $D_{2 h}$ symmetry elements. We note:

$$
\text { (13) }|1 \overline{2} 3 \overline{4}|=|3 \overline{2} 1 \overline{4}|=-|1 \overline{2} 3 \overline{4}| \text {. }
$$

Therefore if $\Psi_{\mathrm{HD}}$ is to contain any $|1 \overline{2} 3 \overline{4}|$ within it it must belong to an irreducible representation which is not the most symmetric representation of $D_{2 h}$. On the other hand, as there are no degenericies in the Hückel molecular orbital, $\Psi_{\text {Hückel }}$ does belong to the most symmetrical representation of $D_{2 h}$. Therefore a crossing is inevitable.

\section{Spin multiplicitles and unsaturated hydrocarbons}

Although our understanding of the electronic structure of unsaturated hydrocarbons is based primarily on Hückel theory, it has been known for some time that Hückel theory is not useful in predicting the spin multiplicity of the ground state of molecules such as cyclobutadiene $\mathbf{1 0}$ and trimethylenemethane 1. It appears in most cases that these systems obey the following simple rule:

$$
S=\left|\frac{N^{*}-N}{2}\right|,
$$

where $S$ is the total spin quantum number of the ground state, $N^{*}$ is the number of starred atoms, and $N$ the number of unstarred atoms. It has also been shown that such a rule is a logical consequence of an HD spin Hamiltonian. ${ }^{1,5}$

The fact that both Hückel and HD Hamiltonians are

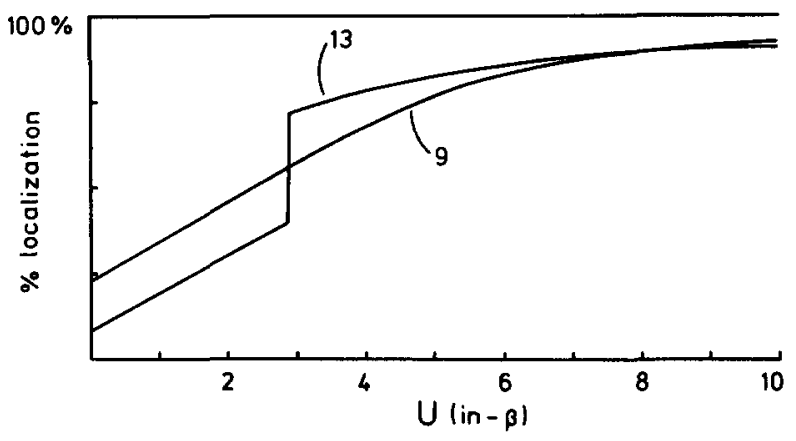

FIG. 4. Electron localization as a function of $|U / \beta|$ for 9 and 13. The curves plotted are $\left\langle\Psi_{\text {Hubbard }}|P| \Psi_{\text {Hubburd }}\right\rangle$ as a function of $U / \beta .13$ shows a discontinuous transition at $U=-2.795 \beta, 9$ does not. Even at $U=-10 \beta$, $\psi_{\text {Hubburd }}$ is only $90 \%$ localized. 
a

$b$

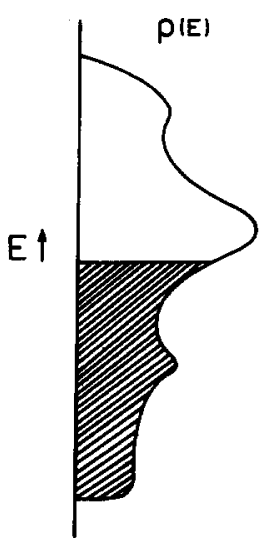

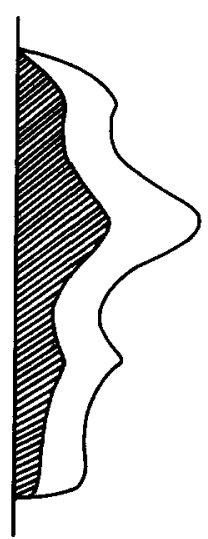

C

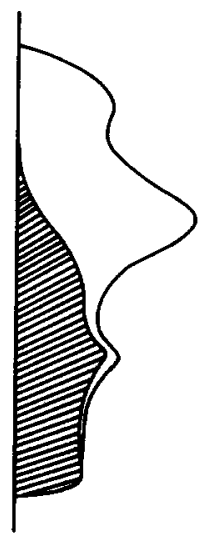

FIG. 5. (a) The band filling of a metal, (b) the traditional view of the band filling of an AF insulator, (c) the intermediate band filling where $U$ is of the same order as band width $W$. It should be noted that $W \simeq-2 m \beta$, where $m$ is the average coordination number. In (c) we have taken a case where $U$ is smaller than $W$.

pertinent to the same set of compounds (i.e., the unsaturated hydrocarbons) seems peculiar, as the two Hamiltonians appear to be based on such different physical interactions.

As has been well established previously ${ }^{1}$ and as has been shown in the previous sections, the reason for this concurrence of results is due to the continuous changeover from the Hückel to HD limits for the case of alternant hydrocarbons. It is therefore not that one of the two Hamiltonians more correctly accounts for the physical forces, but that both Hamiltonians are appropriate zeroth order models.

Thus one may equally well use Hückel theory as a starting point. ${ }^{8}$ In this case one treats $U$ as the perturbation. First order perturbation theory correctly places the triplet in the ground state of $\mathbf{1}$. For $\mathbf{1 0}$, one must resort to second order theory.

\section{Extended systems}

In the case of infinite systems, the changeover from the low spin configuration where electrons are localized to the low spin configuration where they are delocalized is termed an antiferromagnetic insulator-metal transition. The traditional view ${ }^{9}$ of these two forms of matter is that shown in Fig. 5. This traditional view implies the changeover occurs in a discontinuous manner, like that we saw in Fig. 4.

It is actually well known ${ }^{10}$ that for the simplest of all infinite systems, i.e., the infinite chain, the Hubbard transition is of a continuous nature. It is generally believed ${ }^{6}$ that the continuous transition is due to the low dimensionality of the one-dimensional system. As we have shown in this paper, of equal if not greater importance is the presence or absence of odd-member rings. We have shown that even finite systems of the tiniest sort which contain odd member rings, indeed do undergo true transitions.

It would be informative to study higher dimensional solids, in which there are no odd member rings and high homogeneity so as to determine whether there is a Hubbard phase transition. One recent numerical study of the square lattice indicates no such phase transition is to be found. ${ }^{11}$ This again

confirms the utility of our intuition garnered from the study of small systems. Another excellent candidate for study is the graphite sheet 14 .

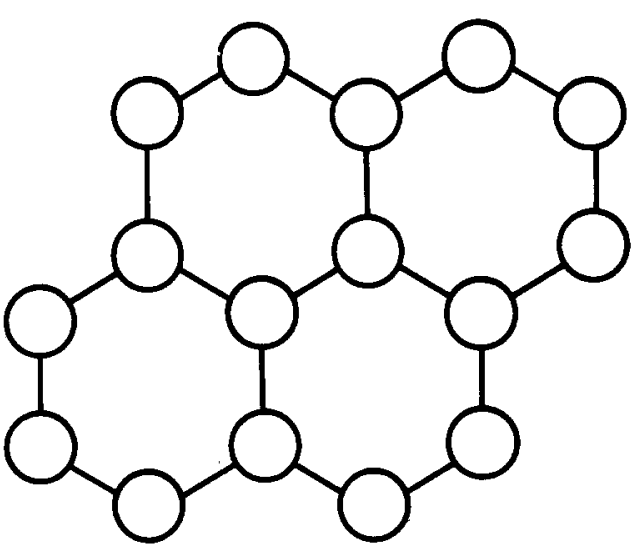

14

\section{ACKNOWLEDGMENTS}

I would like to thank Professor J. Burdett, Professor J. Rouxel, and Professor W. Jeitschko for their assistance. The research was possible due to grants from the NATO postdoctoral program, the CNRS of France, and the A. von Humboldt fellowship program.

'(a) D. J. Klein, C. J. Nelin, S. Alexander, and F. A. Matsen, J. Chem. Phys. 77, 3101 (1982); (b) D. J. Klein, J. Chem. Phys. 77, 3098 (1982). ${ }^{2}$ (a) J. Hubbard, Proc. R. Soc. London Ser. A 276, 238 (1963); 829, 5410 (1984); (b) H. Shiba and P. Pincus, Phys. Rev. B 5, 1966 (1972); (c) D. R. Herrick, J. Chem. Phys. 74,1239 (1981); D. R. Herrick and C.-L. Liao, J. Chem. Phys. 75, 4485 (1981); (e) D. R. Herrick, Adv. Chem. Phys. 52, 1 (1983); (f) F. A. Matsen and T. L. Welsher, Int. J. Quantum Chem. 12, 985, 1001 (1977); (g) F. A. Matsen, Int. J. Quantum Chem. 43, 185 (1984). Also, it should be noted that the HD Hamiltonian arises asymptotically from the Hubbard Hamiltonian in second-order preturbation where one considers the limit $U / \beta>1$.

${ }^{3}$ C. A. Coulson and G. S. Rushbrooke, Proc. Chambridge Philos. Soc. 36, 193 (1940).

${ }^{4} J$. K. Burdett and S. Lee, J. Am. Chem. Soc. 107, 3050 (1985).

${ }^{5}$ (a) E. Lieb, T. Schultz, and D. Mattis, Ann. Phys. 16, 407 (1961); (b) E. Lieb and D. Mattis, J. Math. Phys. 3, 749 (1962).

${ }^{6}$ But see discussion on the errors involved in P. W. Anderson, Basic Notions of Condensed Matter of Physics (Benjamin, Menlo Park, CA), pp. 125129.

${ }^{7}$ For a review of the effective Hamiltonian method, see P. J. Ellis and E. Osnes, Rev. Mod. Phys. 49, 777 (1977). A detailed analysis of thie higher order corrections to the spin Hamiltonian is given in J. P. Malrieu and D. Maynau, J. Am. Chem. Soc. 104, 3021,3029 (1982).

${ }^{8}$ W. T. Borden and E. R. Davidson, J. Am. Chem. Soc. 99, 4587 (1977).

${ }^{9}$ (a) M. W. Whangbo, in Extended Linear Chain Compounds, Vol. 2, edited by J. S. Miller (Plenum, New York, 1982); (b) M. H. Whangbo, in Crystal Chemistry and Properties of Materials with Quasi One Dimensional Structures, edited by J. Rouxel (Reidel, Dordrecht, 1986).

${ }^{10}$ E. H. Lieb and F. Y. Wu, Phys. Rev. Lett. 20, 1445 (1986).

11J. E. Hirsch, Rev. Phys. B 31, 4403 (1985). 